\title{
Computed tomography-measured bone mineral density as a surrogate marker of survival after resection of colorectal liver metastases
}

\author{
Shinichi Ikuta^, Tsukasa Aihara, Takayoshi Nakajima, Meidai Kasai, Naoki Yamanaka \\ Department of Surgery, Meiwa Hospital, Nishinomiya, Hyogo, Japan \\ Contributions: (I) Conception and design: S Ikuta; (II) Administrative support: N Yamanaka; (III) Provision of study material or patients: All authors; \\ (IV) Collection and assembly of data: S Ikuta; (V) Data analysis and interpretation: S Ikuta; (VI) Manuscript writing: All authors; (VII) Final approval \\ of manuscript: All authors. \\ Correspondence to: Shinichi Ikuta. Department of Surgery, Meiwa Hospital, Agenaruo 4-31, Nishinomiya, Hyogo 663-8186, Japan. \\ Email: g2s1002@gmail.com.
}

Background: Osteopenia/osteoporosis, characterized by low bone mineral density (BMD), is a potential
prognostic factor in cancer patients. We conducted a retrospective single-institution study to evaluate the
prognostic impact of preoperative low BMD on colorectal liver metastases (CRLM) in patients undergoing
liver resection.

Methods: BMD was assessed in 281 patients undergoing initial liver resection for CRLM by analyzing the preoperative computed tomography (CT) images at the level of the eleventh thoracic vertebra as the region of interest. Survival outcomes were compared between the two groups divided by the median BMD value and prognostic factors after surgery were assessed. Propensity score-based inverse probability weighting (IPW) was applied to adjust for between-group differences in baseline characteristics.

Results: The low BMD group had significantly more older patients ( $\geq 75$ years) $(\mathrm{P}=0.01)$ and a higher incidence of bilobar metastases $(\mathrm{P}=0.005)$ than the normal BMD group. After IPW adjustment, overall survival (OS) was significantly poorer $(\mathrm{P}=0.02)$ and recurrence-free survival was slightly poorer $(\mathrm{P}=0.05)$ in the low BMD group than in the normal BMD group. IPW-adjusted regression analysis revealed that low $\mathrm{BMD}$ was independently associated with an adverse OS (hazard ratio, 1.42; 95\% CI, 1.04-1.93; $\mathrm{P}=0.03$ ), in addition to other factors such as tumor number, extrahepatic disease, preoperative carcinoembryonic antigen level ( $\geq 5 \mathrm{ng} / \mathrm{mL}$ ), and right-sided primary tumor location.

Conclusions: Preoperative CT-measured low BMD can serve as a surrogate marker of adverse OS in CRLM patients undergoing liver resection. Prevention and early intervention for osteopenia/osteoporosis may be suggested for these patients.

Keywords: Colorectal liver metastases (CRLM); bone mineral density (BMD); osteopenia; osteoporosis

Submitted May 06, 2020. Accepted for publication Sep 30, 2020.

doi: 10.21037/atm-20-3751

View this article at: http://dx.doi.org/10.21037/atm-20-3751

\section{Introduction}

Colorectal cancer (CRC) is one of the most common human malignancies, being the second leading cause of cancer death worldwide (1). About $25 \%$ of CRC patients present with distant metastases at diagnosis, and approximately half of patients will develop liver metastases at some point in their course of disease (2). Over the past several decades,

\footnotetext{
^ ORCID: 0000-0002-9242-8666.
} 
considerable progress has been made in the management of metastatic CRC leading to a significant improvement in 5-year survival. Some of this success has been rightly attributed to aggressive surgical management and advances in other adjunct treatments (3). Treatment options for colorectal liver metastasis (CRLM) include liver resection, coagulation therapy, hepatic arterial infusion chemotherapy, and systemic chemotherapy. Of these, liver resection provides the best chance of cure, with 5-year survival rates of $30-50 \%$ depending upon selection criteria $(4,5)$. Even patients with initially unresectable CRLM can sometimes be cured with multidisciplinary treatment, including staged liver resection, systemic chemotherapy, and targeted therapy (6).

Although the tumor burden, such as tumor size, number, and extrahepatic involvement, is an important determinant of CRLM outcome, patient-level factors may have a significant impact on the prognosis. There is growing evidence that depletion of skeletal muscle mass (sarcopenia) and an increased amount of intra-abdominal fat (central obesity) are associated with the poor outcomes of cancer patients (7-11). Previous studies identified sarcopenia and central obesity as factors adversely influencing survival following resection of CRLM, although there is no consensus in the literature (9-11).

Decreased bone mineral density (BMD) is another important survivorship issue in cancer care, rendering patients at risk for osteoporosis and consequent fractures that can compromise the quality of life and longevity (12). Skeletal complications in cancer patients primarily develop due to bone metastases, but other cancer-related factors as well as aging can cause disturbances in bone remodeling and resultant bone loss $(13,14)$. However, except for cancers that frequently require antihormonal therapies, such as breast cancer and prostate cancer, limited studies have explored the implications of bone loss on outcomes of cancer patients. Previous studies suggested that a low BMD is associated with poorer outcomes after surgical resection among patients with hepatocellular carcinoma (HCC) and extrahepatic biliary cancers $(15,16)$. In addition, low BMD was predictive of mortality in HCC patients undergoing liver transplantation (17).

Our hypothesis was that a preoperative low BMD will negatively affect long-term outcomes after liver resection for CRLM. Thus, the present study examined the impact of $\mathrm{BMD}$, which was estimated using preoperative computed tomography (CT), on survival among resected CRLM patients. We present the following article in accordance with the STROBE reporting checklist (available at http:// dx.doi.org/10.21037/atm-20-3751).

\section{Methods}

\section{Patients, data collection and ethics statement}

Using the prospectively maintained database, we retrospectively enrolled 310 consecutive patients with histopathologically proven CRLM who underwent initial liver resection at our institution between February 28, 2002 and December 31, 2018. Patients with extrahepatic metastases at the time of liver resection, or those treated by liver resection and concomitant ablation were also included. Exclusion criteria included missing important data (e.g., serum levels of tumor markers, size and spread of CRLM), absence of primary tumor resection, and patients without abdominal or chest CT images taken within 3 months before surgery. In addition to the CT measured BMD described below, medical records were reviewed for patient demographics, tumor characteristics, treatment variables, and survival outcomes. The serum tumor marker levels were measured within one month before liver resection using the chemiluminescent enzyme immunoassay kit (Fujirebio, Tokyo, Japan) for carcinoembryonic antigen (CEA) and carbohydrate antigen 19-9. The median follow-up time was estimated using the reverse Kaplan-Meier method. Overall survival (OS) was defined from the date of liver resection to that of death or last contact with the patient (censored). For patients who had no evidence of disease following surgery, recurrence-free survival (RFS) was calculated from the date of liver resection to that of first recurrence at any site. The RFS data was censored for patients alive without tumor recurrence at the last date of follow-up or dead without evidence of tumor recurrence. This study was approved by the institutional ethics committee of Meiwa Hospital (approval No.: 2020-23) for a retrospective analysis of the collected data in accordance with the ethical standards of the World Medical Association's Declaration of Helsinki (as revised in 2013). Individual consent for this retrospective analysis was waived.

\section{Bone mineral density quantification from $C T$}

CT examinations were performed using a multidetectorrow scanner (Brilliance-iCT, Philips Medical Systems, Cleveland $\mathrm{OH}$ ) with scan parameters of $120 \mathrm{kVp} / 200 \mathrm{mAs}$, $128 \times 0.625-\mathrm{mm}$ slice collimation, and $512 \times 512$ pixels. As 

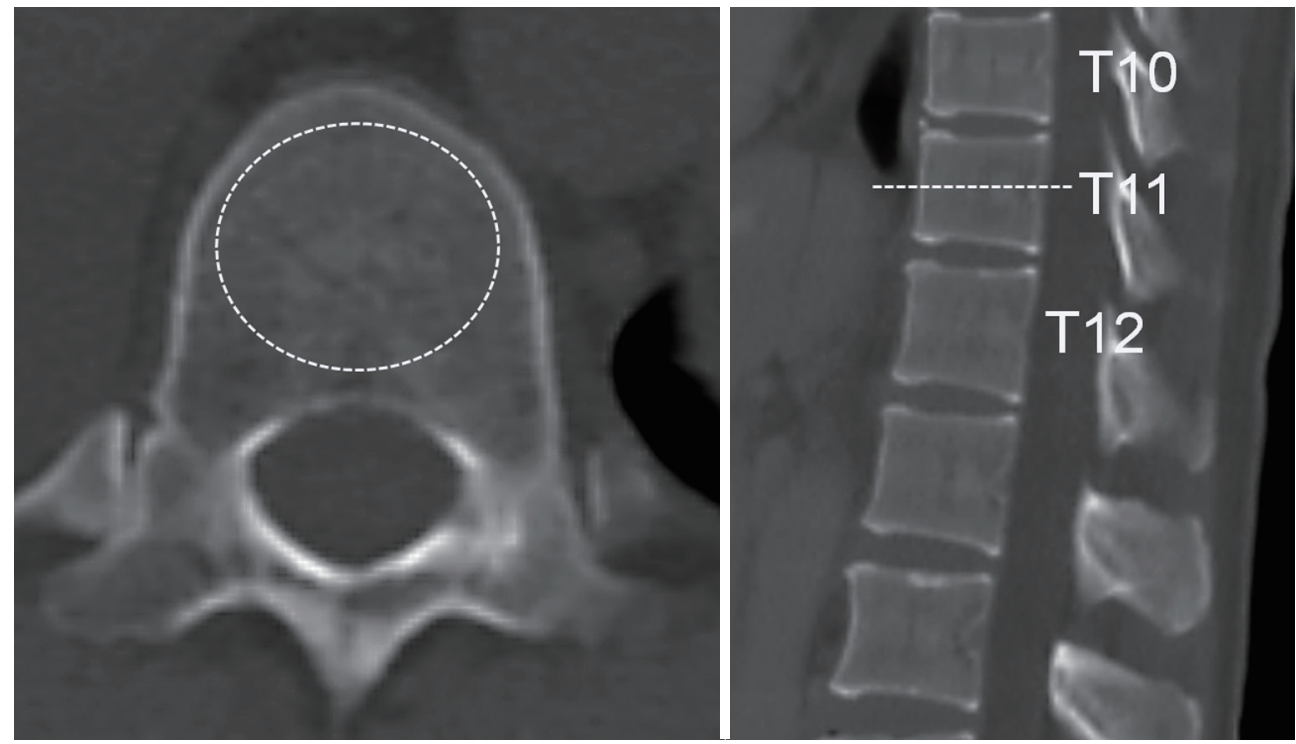

Figure 1 Computed tomography attenuation of the eleventh thoracic vertebrae (T11) was determined by placing a region of interest (dash circle) in the central part of the vertebral body.

previous studies demonstrated a reliable correlation between CT-measured Hounsfield units (HU) and the dual-energy X-ray absorptiometry (DXA)-measured BMD (18-21), axial plain CT images were analyzed using a picture archiving and communication system (ShadeQuest/ViewR V1.22.81, Yokogawa Medical Solutions, Tokyo) for BMD estimation, i.e., CT attenuation values (HU) of the eleventh thoracic vertebrae were measured by placing a circular region of interest on the central part of the vertebral body, as reported previously (17-21). This anatomic landmark was selected because it had the highest likelihood of being available on all abdominal and chest CT images taken in routine practice. The most central section of the vertebra was selected by inspecting the sagittal reformats (Figure 1). We used the median HU as a cutoff to distinguish between normal and low BMD patient groups due to the absence of a widely accepted threshold value for diagnosing osteopenia or osteoporosis.

\section{Statistical analysis}

For between-group comparisons, either the chi-square test or Fisher's exact test was used for categorical variables. To adjust for anticipated baseline characteristics, the two groups (normal BMD vs. low BMD) were balanced by propensity score-based inverse probability weighing (IPW). The propensity score was calculated for each patient using a logistic regression model that included the baseline characteristics. Covariate balance after the IPW was assessed by computing their standardized differences and groups were considered balanced if the standardized differences of all covariates were $<0.1$. IPW-adjusted Kaplan-Meier curves were calculated to compare OS and RFS between patients with a low BMD and those with a normal BMD. Differences were compared using the weighted log rank test. IPW-adjusted Cox regression analyses were performed to identify independent predictors of OS and RFS, and for subgroup analysis. All statistical analyses were performed using R 3.6.1 software (Foundation for Statistical Computing, Vienna, Austria) and $\mathrm{P}<0.05$ was considered significant.

\section{Results}

\section{Baseline clinical characteristics}

After excluding 29 patients who met the exclusion criteria, 281 patients were finally analyzed. The patients consisted of 162 males and 119 females with a median age of 66 years (range, $35-88$ years). The median value of BMD was $141 \mathrm{HU}$ (range, 22-345 HU). One hundred and thirtyeight patients had a normal BMD (BMD $\geq 141 \mathrm{HU})$ and 143 patients had a low BMD (BMD <141 HU). The baseline characteristics compared between the normal and 
Table 1 Baseline patient characteristics

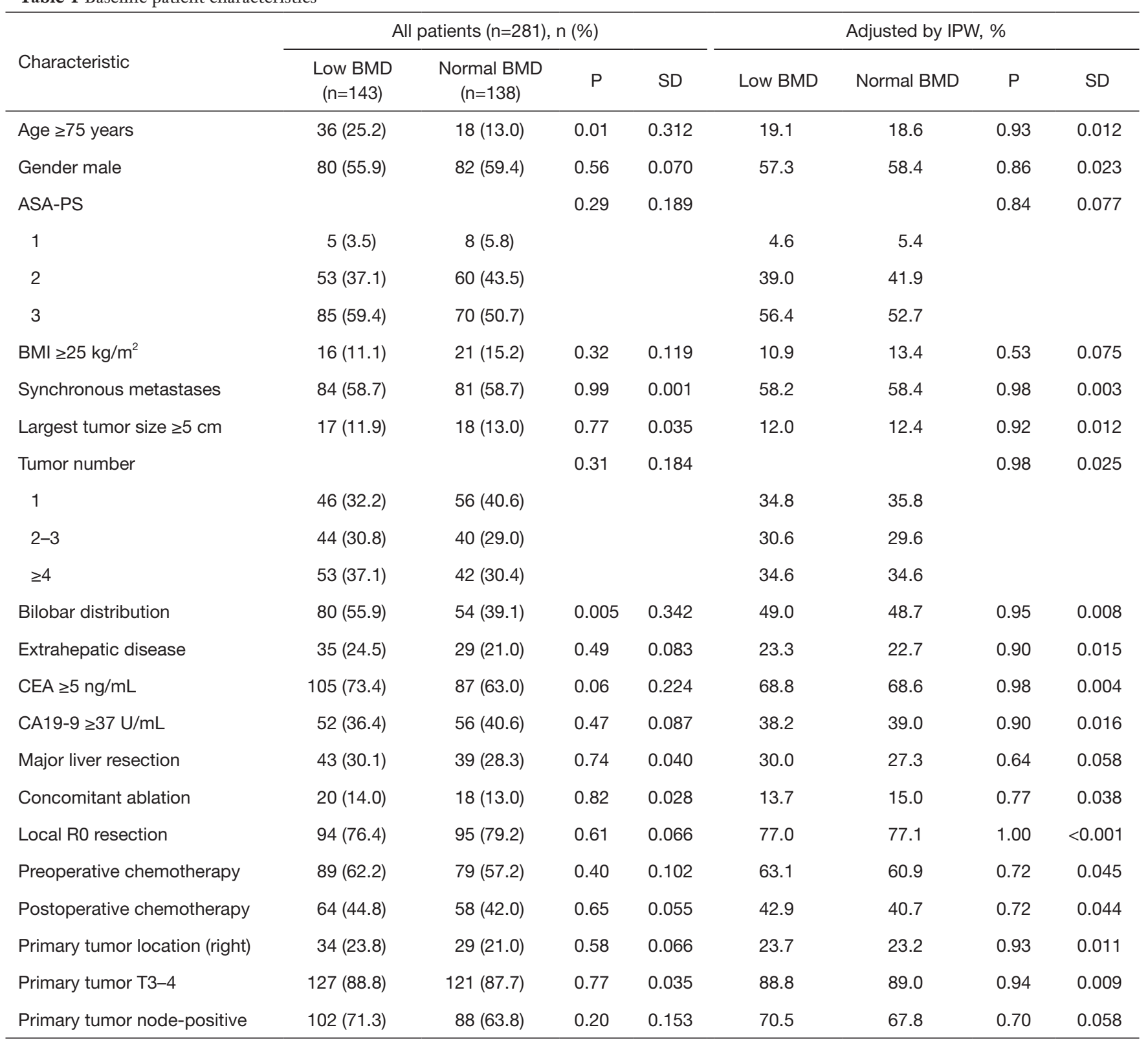

IPW, inverse probability weighing; BMD, bone mineral density; SD, standardized difference; ASA-PS, American Society of Anesthesiologists physical status; BMI, body mass index; CEA, carcinoembryonic antigen; CA19-9, carbohydrate antigen 19-9.

low BMD groups are shown in Table 1. The low BMD group had significantly older ( $\geq 75$ years) patients and those with bilobar metastases than the normal BMD group. Moreover, the low BMD group had a slightly higher CEA level $(\geq 5 \mathrm{ng} / \mathrm{mL}$ ) than the normal BMD group, but this was not significant. After application of IPW, the characteristics of interest were well balanced with a standard difference of less than 0.1 .

\section{Kaplan-Meier survival analysis, and univariate and multivariate Cox analyses}

The median follow-up in the weighted population was 64.6 months [95\% confidence interval (CI): 60.466.8 months]. Based on IPW-adjusted Kaplan-Meier curves (Figure 2), the median OS was significantly shorter in the low BMD group than in the normal BMD group 

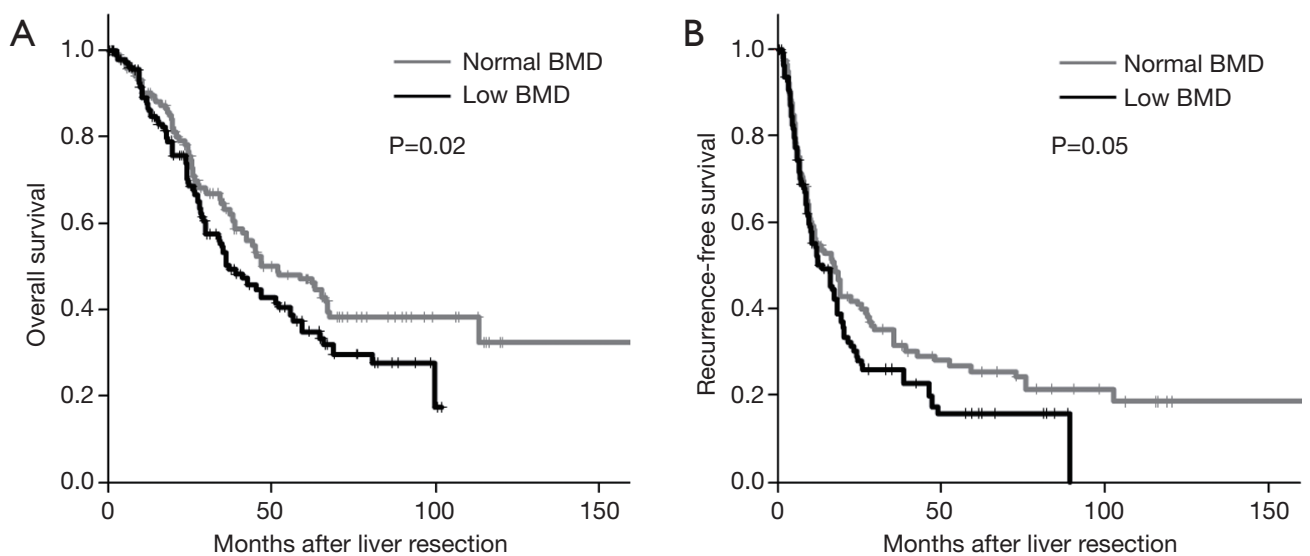

Figure 2 Inverse probability weighting-adjusted Kaplan-Meier analysis of overall survival (A) and recurrence-free survival (B) for the low versus normal BMD groups. BMD, bone mineral density.

[36.7 (95\% CI: 33.8-46.7) vs. 51.7 (95\% CI: 42.1-65.0) months; $\mathrm{P}=0.02]$. The multivariate adjusted Cox model demonstrated a low BMD to be independently associated with an adverse OS [hazard ratio (HR), 1.42 (95\% CI: 1.041.93); $\mathrm{P}=0.03$ ], in addition to other factors listed in Table 2 . When 77 patients with local R2 resection $(\mathrm{n}=18)$ and/or extrahepatic disease at hepatectomy $(n=64)$ were excluded, the median RFS in the low BMD group ( $\mathrm{n}=101)$ was slightly shorter than that in the normal BMD group $(n=103)[13.4$ (95\% CI: 10.1-17.1) vs. 17.0 (95\% CI: $11.0-18.9)$ months], but the difference was of borderline significance $(\mathrm{P}=0.05)$ (Figure 2). Lower BMD lost significance as a predictor of RFS in multivariate analysis [HR, 1.26 (95\% CI: 0.95-1.67); $\mathrm{P}=0.11$ ] (Table 2). The subgroup analysis stratified by age, gender, and American Society of Anesthesiologists physical status was performed using adjusted Cox model to further explore the association between BMD and OS. As shown in Figure 3, low BMD tended to be consistently associated with worse OS, although not all met statistical significance.

\section{Discussion}

Osteopenia or osteoporosis with advancing age is a major public health challenge worldwide. In Western countries, the lifetime risk of osteoporotic fracture remains high, within the range of $40-50 \%$ for women and $13-22 \%$ for men (22). DXA is currently the standard for assessing $\mathrm{BMD}$, and is correlated with fracture risk and treatment efficacy (23). We instead calculated vertebral body CT HU values using routinely obtained images in preoperative evaluations, as findings of multiple studies have suggested that this method can provide surrogate information on BMD without the need for additional costs or radiation exposure (18-21). A strong correlation between CT HU values and DXA T-scores, which is used by the World Health Organization criteria for diagnosis of osteopenia/ osteoporosis, was previously reported $(20,21)$. In an analysis of 109 patients who underwent both DXA and CT for unrelated reasons, Li et al. reported that mean vertebral CT HU values were 230 (95\% CI: 203-254) for the normal subgroup, 135 (95\% CI: 122-148) for the osteopenia subgroup, and 97 (95\% CI: 88-105) for the osteoporotic subgroup (19). In a study with a large $(n=2,020)$ heterogeneous population of otherwise healthy trauma patients, Patel et al. found that the radiologist-modified lumbar vertebral body $\mathrm{HU}$ corresponding to osteopenia was $139.4 \pm 48.8$ (20), which was relatively close to the threshold of 141 we used to distinguish patients with and without osteopenia/osteoporosis in this study.

To our knowledge, this is the first study to propose the novel association between CT-measured BMD and adverse OS among CRLM patients undergoing liver resection. One possible explanation for our results is that a preoperative low BMD somewhat reflects a high tumor burden. Loss of extracellular calcium-sensing receptor expression is strongly linked to CRC progression and parathyroid hormone (PTH)-induced bone loss $(24,25)$. Although uncommon, increased resorption and accelerated turnover of bone mediated by the systemic secretion of PTH-related protein should be considered in patients with metastatic CRC presenting with hypercalcemia (26). In addition, sarcopenia, which is partly explained by metabolic demands 
Table 2 IPW-adjusted univariate and multivariate analyses of prognostic factors

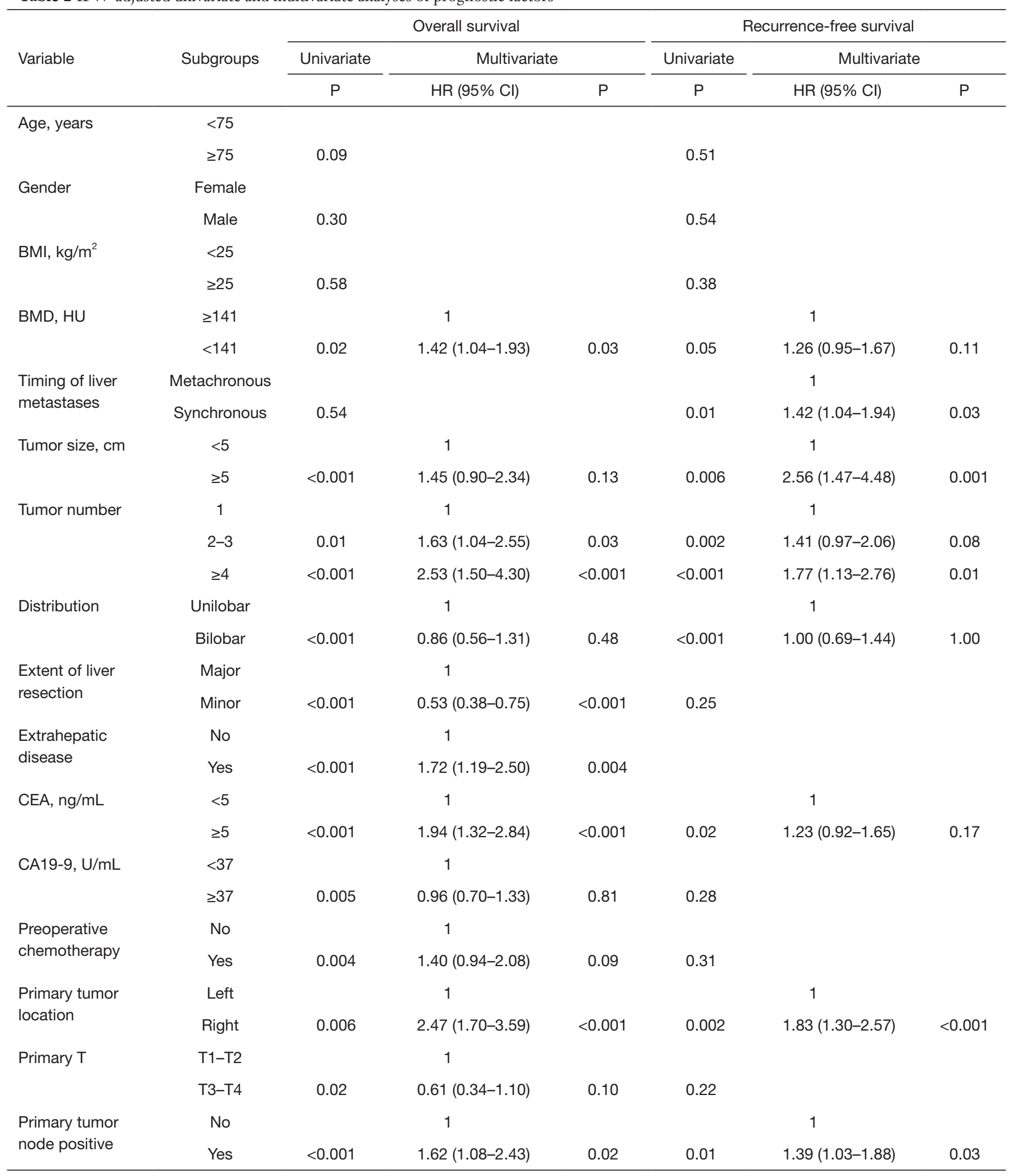

IPW, inverse probability weighing; Cl, confidence interval; BMI, body mass index; BMD, bone mineral density; HU, Hounsfield units; CEA, carcinoembryonic antigen; CA19-9, carbohydrate antigen 19-9. 


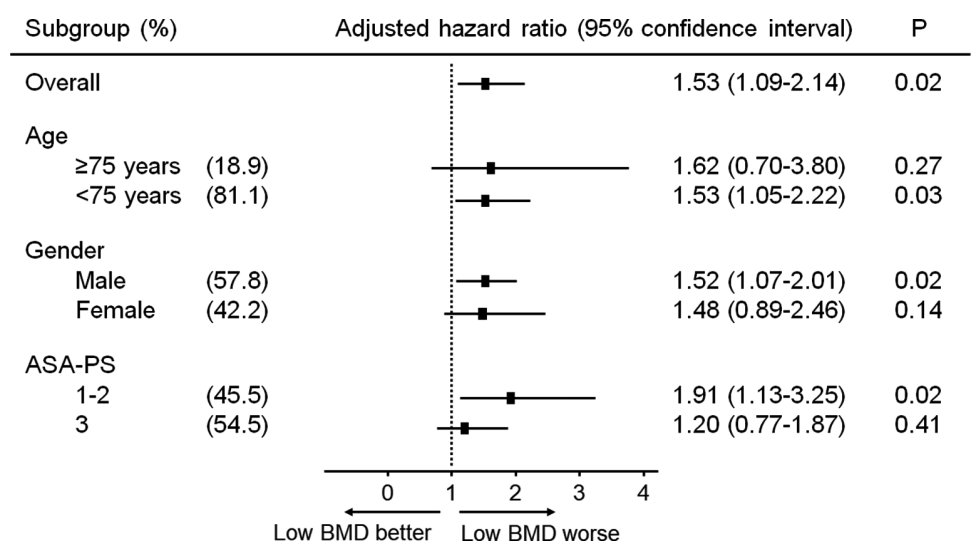

Figure 3 Forest plot of subgroup analysis for overall survival. ASA-PS, American Society of Anesthesiologists physical status; BMD, bone mineral density.

from a high tumor burden, also exhibited a significant association with osteopenia/osteoporosis in previous studies and can have a negative impact on outcomes concerning RFS and OS following resection of CRLM (9,27-29). The regression analyses by Pereira et al. demonstrated that elderly men diagnosed with pre-sarcopenia and sarcopenia had a more abnormal BMD than non-sarcopenic elderly men (28). Another study by Lima et al. found a doseresponse relationship among sarcopenia stages, BMD, and the presence of osteoporosis in older women (29). Recent studies suggest that bone loss begins before the loss of muscle mass, and thus may be an early marker of deconditioning that precedes sarcopenia in patients with end-stage liver disease $(17,28)$.

Cancer chemotherapies further accelerate bone loss through direct dysregulation of bone turnover and indirect mechanisms such as nephrotoxicity $(14,30,31)$. Such therapies include antineoplastic drugs, such as platinumderived compounds, alkylating agents, antimetabolites, glucocorticoids, and targeted therapies. The combination therapy Folfiri (5-fluorouracil, leucovorin, and irinotecan) also reduces the bone volume (32). Indeed, in our unweighted population, the low BMD group had a higher rate of having aggressive clinicopathologic features than the normal BMD group, i.e., tumor number, CEA level, extrahepatic disease, primary tumor nodal status, and preoperative chemotherapy, although most were not significant.

However, in the present study, a low BMD was an independent factor for an adverse OS, but of borderline significance for RFS after covariate adjustment using IPW. Another possible explanation for our finding is that low BMD-specific outcomes, particularly frailty fractures, may have a significant negative impact on physical activity and functional status, leading to nonadherence or discontinuation of cancer therapy, or to non-cancer mortality. Compared with men who had maintained BMD, those who had accelerated BMD loss had a $44 \%$ greater risk of mortality among 4,400 Osteoporotic Fractures in Men (MrOS) study participants (33). Approximately 50\% of patients who sustain a hip fracture lose the ability to walk independently and the reported 1-year mortality after sustaining a hip fracture has been estimated to be $14 \%$ to $58 \%(34,35)$. McDonald et al. reported in their retrospective study that age-adjusted CT attenuation of L5 vertebrae was an independent predictor of non-cancer death in men with prostate cancer (36). Our finding that BMD is associated with OS may be related not only to the tumor, but also to host pathology.

The major limitations of the study are inherent biases associated with its retrospective design and low power due to the limited sample size, potentially leading to the loss of statistical significance in some of results, especially in subgroup analysis. Another important limitation is that changes in BMD over time, osteopenia/osteoporosisspecific outcomes, and their therapies were not assessed in this study, and their relevance with the survival of patients remains to be clarified. Third, our study did not evaluate lifestyles that may affect BMD such as dietary choices, exogeneous hormone use, smoking, alcohol consumption, and physical activity.

In summary, CT-measured BMD, which can be easily obtained from pre-operative routine examinations, can be a surrogate biomarker for OS in patients with CRLM undergoing liver resection. Although a further prospective 
large-scale study to validate the study findings is needed, our results support the importance of prevention and early intervention for osteopenia/osteoporosis due to aging, cancer progression, and its therapies in these patients.

\section{Acknowledgments}

Funding: None.

\section{Footnote}

Reporting Checklist: The authors have completed the STROBE reporting checklist. Available at http://dx.doi. org/10.21037/atm-20-3751

Data Sharing Statement: Available at http://dx.doi. org/10.21037/atm-20-3751

Peer Review File: Available at http://dx.doi.org/10.21037/ atm-20-3751

Conflicts of Interest: All authors have completed the ICMJE uniform disclosure form (available at http://dx.doi. org/10.21037/atm-20-3751). The authors have no conflicts of interest to declare.

Ethical Statement: The authors are accountable for all aspects of the work in ensuring that questions related to the accuracy or integrity of any part of the work are appropriately investigated and resolved. The study was conducted in accordance with the Declaration of Helsinki (as revised in 2013). The study was approved by institutional ethics committee of Meiwa Hospital (approval No.: 2020-23) and individual consent for this retrospective analysis was waived.

Open Access Statement: This is an Open Access article distributed in accordance with the Creative Commons Attribution-NonCommercial-NoDerivs 4.0 International License (CC BY-NC-ND 4.0), which permits the noncommercial replication and distribution of the article with the strict proviso that no changes or edits are made and the original work is properly cited (including links to both the formal publication through the relevant DOI and the license). See: https://creativecommons.org/licenses/by-nc-nd/4.0/.

\section{References}

1. Bray F, Ferlay J, Soerjomataram I, et al. Global cancer statistics 2018: GLOBOCAN estimates of incidence and mortality worldwide for 36 cancers in 185 countries. CA Cancer J Clin 2018;68:394-424.

2. Adam R, De Gramont A, Figueras J, et al. The oncosurgery approach to managing liver metastases from colorectal cancer: a multidisciplinary international consensus. Oncologist 2012;17:1225-39.

3. Pai SG, Fuloria J. Novel therapeutic agents in the treatment of metastatic colorectal cancer. World J Gastrointest Oncol 2016;8:99-104.

4. Malik HZ, Prasad KR, Halazun KJ, et al. Preoperative prognostic score for predicting survival after hepatic resection for colorectal liver metastases. Ann Surg 2007;246:806-14.

5. Simmonds PC, Primrose JN, Colquitt JL, et al. Surgical resection of hepatic metastases from colorectal cancer: a systematic review of published studies. Br J Cancer 2006;94:982-99.

6. Popescu I, Alexandrescu S, Croitoru A, et al. Strategies to convert to resectability the initially unresectable colorectal liver metastases. Hepatogastroenterology 2009;56:739-44.

7. Kobayashi A, Kaido T, Hamaguchi Y, et al. Impact of sarcopenic obesity on outcomes in patients undergoing hepatectomy for hepatocellular carcinoma. Ann Surg 2019;269:924-31.

8. Harimoto N, Shirabe K, Yamashita YI, et al. Sarcopenia as a predictor of prognosis in patients following hepatectomy for hepatocellular carcinoma. Br J Surg 2013;100:1523-30.

9. van Vledder MG, Levolger S, Ayez N, et al. Body composition and outcome in patients undergoing resection of colorectal liver metastases. Br J Surg 2012;99:550-7.

10. Peng PD, van Vledder MG, Tsai S, et al. Sarcopenia negatively impacts short-term outcomes in patients undergoing hepatic resection for colorectal liver metastasis. HPB (Oxford) 2011;13:439-46.

11. Lodewick TM, van Nijnatten TJ, van Dam RM, et al. Are sarcopenia, obesity and sarcopenic obesity predictive of outcome in patients with colorectal liver metastases? HPB (Oxford) 2015;17:438-46.

12. Taxel P, Faircloth E, Idrees S, et al. Cancer treatmentinduced bone loss in women with breast cancer and men with prostate cancer. J Endocr Soc 2018;2:574-88.

13. Coleman R, Body JJ, Aapro M, et al. Bone health in cancer patients: ESMO Clinical Practice Guidelines. Ann Oncol 2014;25 Suppl 3:iii124-37.

14. Dolly A, Lecomte T, Bouché O, et al. Concurrent losses of skeletal muscle mass, adipose tissue and bone mineral density during bevacizumab / cytotoxic chemotherapy 
treatment for metastatic colorectal cancer. Clin Nutr 2020;S0261-5614:30080-7.

15. Miyachi Y, Kaido T, Yao S, et al. Bone mineral density as a risk factor for patients undergoing surgery for hepatocellular carcinoma. World J Surg 2019;43:920-8.

16. Yao S, Kaido T, Okumura S, et al. Bone mineral density correlates with survival after resection of extrahepatic biliary malignancies. Clin Nutr 2019;38:2770-7.

17. Sharma P, Parikh ND, Yu J, et al. Bone mineral density predicts posttransplant survival among hepatocellular carcinoma liver transplant recipients. Liver Transpl 2016;22:1092-8.

18. Schreiber JJ, Anderson PA, Hsu WK. Use of computed tomography for assessing bone mineral density. Neurosurg Focus 2014;37:E4.

19. Li YL, Wong KH, Law MW, et al. Opportunistic screening for osteoporosis in abdominal computed tomography for Chinese population. Arch Osteoporos 2018;13:76.

20. Patel SP, Lee JJ, Hecht GG, et al. Normative vertebral hounsfield unit values and correlation with bone mineral density. J Clin Exp Orthop 2016;2:14.

21. Schreiber JJ, Anderson PA, Rosas HG, et al. Hounsfield units for assessing bone mineral density and strength: a tool for osteoporosis management. J Bone Joint Surg Am 2011;93:1057-63.

22. Johnell O, Kanis J. Epidemiology of osteoporotic fractures. Osteoporos Int 2005;16 Suppl 2:S3-7.

23. Marshall D, Johnell O, Wedel H. Meta-analysis of how well measures of bone mineral density predict occurrence of osteoporotic fractures. BMJ 1996;312:1254-9.

24. Iamartino L, Elajnaf T, Kallay E, et al. Calcium-sensing receptor in colorectal inflammation and cancer: current insights and future perspectives. World J Gastroenterol 2018;24:4119-31.

25. Momen-Heravi F, Masugi Y, Qian ZR, et al. Tumor expression of calcium sensing receptor and colorectal cancer survival: results from the nurses' health study and health professionals follow-up study. Int J Cancer

Cite this article as: Ikuta S, Aihara T, Nakajima T, Kasai M, Yamanaka N. Computed tomography-measured bone mineral density as a surrogate marker of survival after resection of colorectal liver metastases. Ann Transl Med 2021;9(1):21. doi: 10.21037/atm-20-3751
2017;141:2471-9.

26. Galindo RJ, Romao I, Valsamis A, et al. Hypercalcemia of malignancy and colorectal cancer. World J Oncol 2016;7:5-12.

27. Verschueren S, Gielen E, O'Neill TW, et al. Sarcopenia and its relationship with bone mineral density in middleaged and elderly European men. Osteoporos Int 2013;24:87-98.

28. Pereira FB, Leite AF, de Paula AP. Relationship between pre-sarcopenia, sarcopenia and bone mineral density in elderly men. Arch Endocrinol Metab 2015;59:59-65.

29. Lima RM, de Oliveira RJ, Raposo R, et al. Stages of sarcopenia, bone mineral density, and the prevalence of osteoporosis in older women. Arch Osteoporos 2019;14:38.

30. Sturgeon KM, Mathis KM, Rogers CJ, et al. Cancerand chemotherapy-Induced musculoskeletal degradation. JBMR Plus 2019;3:e10187.

31. D'Oronzo S, Stucci S, Tucci M, et al. Cancer treatmentinduced bone loss (CTIBL): pathogenesis and clinical implications. Cancer Treat Rev 2015;41:798-808.

32. Barreto R, Waning DL, Gao H, et al. Chemotherapyrelated cachexia is associated with mitochondrial depletion and the activation of ERK1/2 and p38 MAPKs. Oncotarget 2016;7:43442-60.

33. Cawthon PM, Patel S, Ewing SK, et al. Bone loss at the hip and subsequent mortality in older men: the osteoporotic fractures in men (MrOS) study. JBMR Plus 2017;1:31-5.

34. Khazzani H, Allali F, Bennani L, et al. The relationship between physical performance measures, bone mineral density, falls, and the risk of peripheral fracture: a crosssectional analysis. BMC Public Health 2009;9:297.

35. Schnell S, Friedman SM, Mendelson DA, et al. The 1-year mortality of patients treated in a hip fracture program for elders. Geriatr Orthop Surg Rehabil 2010;1:6-14.

36. McDonald AM, Swain TA, Mayhew DL, et al. CT measures of bone mineral density and muscle mass can be used to predict noncancer death in men with prostate cancer. Radiology 2017;282:475-83. 